\title{
PROYECTOS DE INVERSIÓN QUE MITIGAN EL IMPACTO AMBIENTAL
}

\author{
Por Nelson Cerda
}

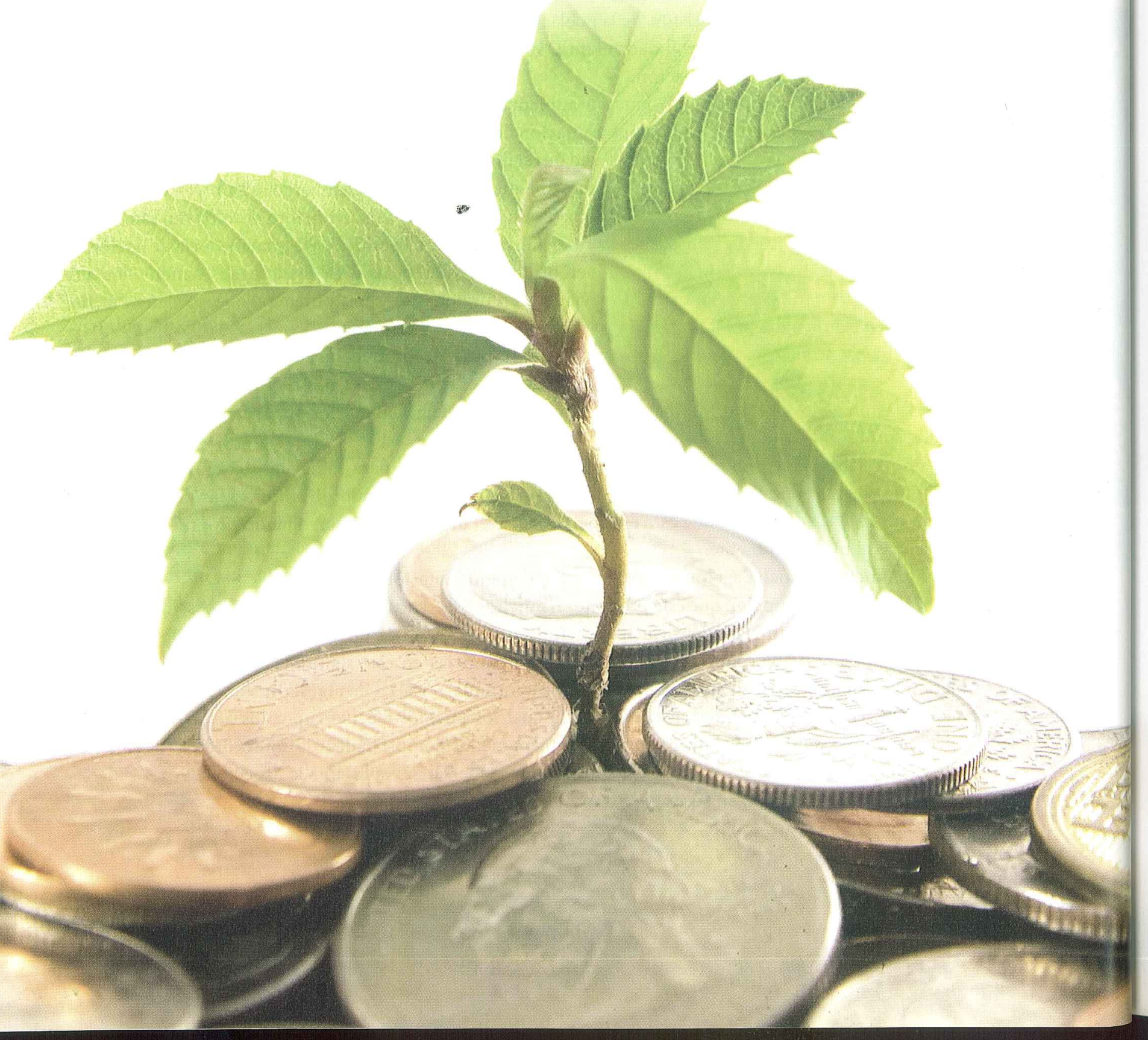


Es indiscutible la necesidad de poner sobre el tapete de discusión, el impacto ambiental que generamos los seres humanos a nuestro planeta, es así que toma mucha fuerza este análisis a nivel mundial, desde el 11 de diciembre de 1997 cuando en Japón fue el inicio de "El Protocolo de Kioto sobre el Cambio Climático", que entró en vigencia el 16 de enero del 2005 y para noviembre de 2009 ya eran más de 100 estados que lo ratificaban.

\section{Pero dQué es el Protocolo de IKyoto Sobre el Cambio Climático?}

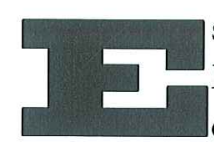
S un Protocolo de la Convención Marco de las Naciones Unidas sobre el Cambio Climático y un acuerdo internacional que se sustenta en la reducción de la emisión de gases que causan el efecto invernadero y por ende el calentamiento global, estos gases son los siguientes: gas metano ( $\mathrm{CH} 4)$, dióxido de carbono (CO2) y óxido nitreso (N2O), adicionalmente tres gases industriales fluorados: hidrofluorocarbonos (HFC), perfluroarbonos (PFC) y hexafluoruro de azufre (SF6).

En tal sentido se establece que para el año 2012 se reducirá aproximadamente el 5\% de emisiones de los gases antes mencionados, analizando las emisiones del año 2009.

El cambio climático a más de afectar o alterar el planeta que involucra grandes daños ecológicos, a través de

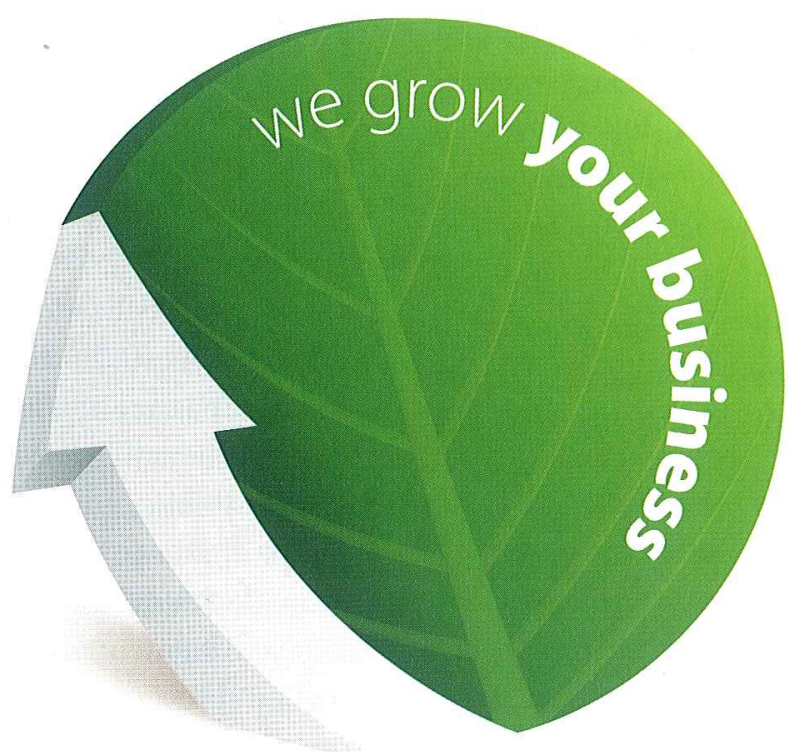

urgentes de mitigación de impacto ambiental.

Es así, que se establecen grandes proyectos estratégicos, que los países pretenden elaborar, evaluar y ejecutar, sin que ello signifique que estas medidas no se conviertan en negocios rentables, pero siempre bajo la premisa de mitigación del impacto ambiental.

La ejecución de los proyectos descritos, pueden ser fuente de ingresos alternos, a través de la emisión de bonos de carbono, mejoramiento en los niveles de productividad, ahorros en costos, etc.

\section{Los bonos de carpono}

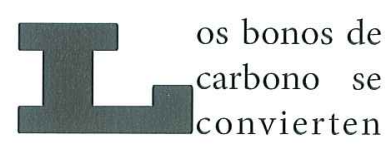
en una herramienta creada para procesos de descontaminación ambiental, a través de la ejecución del Mecanismo de Desarrollo Limpio "MDL" que permite la disminución de emisión de gases que causan el efecto invernadero y por ende el calentamiento global del planeta.

Los bonos de carbono, es un sistema de incentivos que permiten a las empresas obtener réditos económicos, a través de la ejecución de proyectos verdes (generación de energías limpias, proyectos forestales y reforestales, procesos de reciclaje, tratamiento de desechos a través de biodigestores, as pérdidas, posteriores a efectos nat agudos, lo que conlleva a plantear medidas 
etc.) y que pueden ser negociados en el mercado a un precio establecido.

Los proyectos antes mencionados, pueden ser certificados bajo un proceso de control riguroso, el cual incluye determinar los beneficios al medio ambiente, a través de reducción de gases de efecto invernadero, efectos que deben ser reales y medibles.

Una vez que los proyectos han sido certificados y pueden emitir bonos de carbono, los mismos serán ofertados al mercado, para que empresas o países que generen actividad contaminante (emisiones de gases de efecto invernadero) puedan adquirirlos y de esta manera reducir el impacto ambiental al planeta.

\section{ITegocios rentables que respetan el medio ambiente}

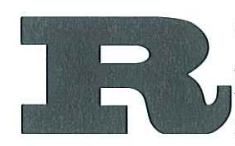
espetar el medio ambiente, por principio ya es un sinónimo de rentabilidad, reflejado en tratar de garantizar un planeta estable para las nuevas generaciones, lo cual por si solo habla de la responsabilidad social de las empresas y de todos los actores en los procesos de la actividad económica en general.

Es así que en la actualidad los proyectos amigables con el medio ambiente, tales como, forestación y reforestación, reciclaje, generación de energías limpias, sistemas de tratamiento de aguas negras, a más de ser una necesidad emergente por los antecedentes expuestos, se han convertido en negocios rentables desde el punto de vista social y económico.

\section{Ios projectos de porestacị́n y de rerirorestacinón}

La silvicultura es una actividad económica rentable, que a su vez se la vincula con el desarrollo sostenible y fue considerada como uno de los temas transversales en la Conferencia de Río +20, realizada en junio pasado.

Es así que la FAO (Organización de las Naciones Unidas para la Alimentación y Agricultura) realiza un análisis completo en su edición 2012, de la situación de los bosques a nivel mundial, y su papel en el desarrollo sostenible.

El Director General de la FAO, el 24 de septiembre en Roma, explicó que el éxito de la labor de su Organización para mejorar las vidas de los seres humanos, depende del uso equilibrado de los recursos naturales, involucrando en este análisis la silvicultura.

De acuerdo a datos establecidos por la Naciones Unidas, aproximadamente 350 millones de personas, entre las cuales se incluyen 60 millones de indígenas, dependen de los bosques para su subsistencia y supervivencia.

Es indiscutible la necesidad de realizar un análisis de la situación de los bosques en la región, en tal sentido es importante mencionar que América Latina y El Caribe mantienen una gran cantidad de recursos forestales, es así que poseen aproximadamente 891 millones de hectáreas de bosques, que representa el $22 \%$ de los bosques mundiales.

Brasil, Colombia, Perú, Bolivia y Venezuela, son los países que mantienen el $84 \%$ de los bosques de la región.

En relación a los bosques primarios, en los cuales se encuentra la mayor biodiversidad del planeta, América Latina y El Caribe son los poseedores del $57 \%$, los cuales se hallan en lugares protegidos, sin embargo son amenazados por los procesos de deforestación (para extracción de madera) y explotación de recursos naturales en forma poco conciente.

Una vez realizado un análisis breve de la situación en estudio en la región, es indispensable identificar la realidad de nuestro país, es así que Ecuador de acuerdo a Tony Zúñiga Suárez en su artículo publicado por la FAO menciona que: "El área estimada de bosque nativo es de 11.5 millones de ha. (cerca del $42 \%$ del territorio), de las cuales el 80\% se encuentra en la Amazonía, un 13\% en la Costa y el restante 7\% en la Sierra. El 29\% del área total de bosques naturales del país está declarado como zona de protección (bosques protectores o áreas naturales protegidas), correspondiendo un $50 \%$ a bosques secos tropicales, un $46 \%$ a bosques de estribaciones y un $4 \%$ a manglares".

Es claro indicar que en nuestro país el desarrollo de la actividad forestal y reforestal en los dos 
últimos años ha tomado mucha importancia, tal es así que el plan nacional de forestación establece que para los próximos 20 años se alcance un millón de hectáreas sembradas, de las cuales 750 mil serán para explotación comercial, 150 agroforestales y 100 mil hectáreas destinadas a la protección y conservación de los recursos naturales.

Los objetivos que se pretenden con este plan son:

- Reducir la presión existente sobre los bosques nativos del país (explotación y deforestación).

- Abastecer la demanda nacional y sustituir importaciones.

- Generar producción excedente para la comercialización en mercados externos.

Para alcanzar estos objetivos se ofrecen al mercado líneas de crédito atractivas que establecen períodos de gracia totales dependiendo de las especies forestales y su destino.

\section{¿ILOs projectos forestales son rentables?}

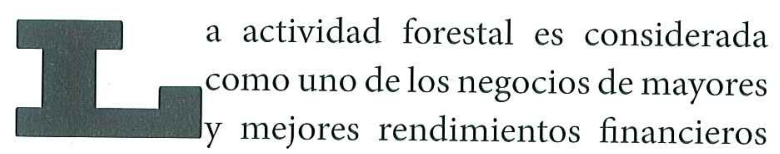
a largo plazo, también se los considera como proyectos de larga maduración.

Se debe considerar que las plantaciones forestales tienen un período pre-operativo largo, es decir que no generan flujo de efectivo durante algunos años, por tal razón los mecanismos financieros y de evaluación que la banca estatal de desarrollo ofrece al mercado, permite que los empresarios puedan iniciar o ampliarse en la actividad forestal, a través de créditos con períodos de gracia totales e intereses razonables.

Las plantaciones forestales durante el avance de la fase vegetativa, también inician un proceso de revaloración monetaria, tal es el caso que transcurrido el 20\% aproximadamente del ciclo normal, las plantas (árboles) tienen un valor superior a la inversión correspondiente a la tierra (terreno).
Los réditos económicos que pueden obtenerse de una plantación forestal, dependiendo de la variedad de la planta, ubicación, etc., son los siguientes:

- Certificación y emisión de bonos de carbono a ser negociados en el mercado internacional.

- Emisión de papel comercial, sustentado en un análisis de rendimientos futuros.

- Compensación por parte del Estado por procesos de forestación y reforestación, a través de bonos financieros que permiten mantener la plantación, generación de flujo de efectivo y su reposición de la plantación posterior a su aprovechamiento comercial.

- Cosecha y procesamiento del proyecto forestal al final del ciclo vegetativo, lo que permitirá la venta de madera, que constituye el ingreso mayor, siempre y cuando el cultivo se lo haya ejecutado en forma técnica y si es posible generando valor agregado al producto, es decir no vender árboles en pie, sino madera procesada (producción de palletes, laminados, glucosa, etc).

\section{Projectos de generacinón de energíns renovables no convencinales}

La generación de energías renovables también conocidas como energías verdes o limpias, son las obtenidas de fuentes primarias por medio de procesos respetuosos del medio ambiente, es así que se consideran dentro de esta calificación proyectos de: energía fotovoltaica, energía a través de biomasa, energía eólica, energía hidráulica, energía geotérmica y energía marina.

Es necesario indicar, que si bien los proyectos antes indicados son conocidos como energías renovables no significan que no generen impacto ambiental, sino que el impacto es menor que los proyectos tradicionales de generación, como es en el uso de combustibles fósiles o a través de energía nuclear, método muy peligroso, que adicionalmente produce desechos nucleares que tardan miles de años en desaparecer su efecto radiactivo.

Existe una discusión permanente en el sentido de catalogar como generación de energía 
limpia a los métodos de biomasa por su proceso de incineración que genera emisiones de $\mathrm{CO} 2$ y las grandes hidroeléctricas que a pesar de utilizar recursos renovables el impacto ambiental es considerable.

En relación a este aspecto la Constitución del Ecuador aprobada en el año 2008 establece que: "El Estado se reserva el derecho a administrar, regular, controlar y gestionar los sectores estratégicos de la economía", en los cuales se incorpora por obvias razones la generación de energía por los medios no convencionales y con recursos renovables.

En la actualidad el CONELEC ha establecido la respectiva normatividad para controlar y ejecutar (que incluye precios/tarifas) proyectos de generación de energías, a través de métodos no convencionales por medio de inversión privada.

$\mathrm{Al}$ ser proyectos de inversión privada en sectores estratégicos del país, es indispensable analizar la seguridad jurídica y obviamente su factibilidad desde el punto de vista financiero (rentabilidad), factores considerados en la normativa vigente en el Ecuador.
La regulación permite la ejecución de proyectos de carácter privado, relacionado a la generación de energías renovables no convencionales, a través de sistemas solares, eólicos, biomasa, biogás e hidroeléctricos considerando siempre que su capacidad máxima instalada no podrá ser superior a $50 \mathrm{MW}$ por proyecto.

Es así, que como cualquier proyecto de inversión, se analiza en función de la regulación vigente, la rentabilidad de los mismos y a través de los diferentes estudios: (mercado, técnico y financiero).

En tal sentido, estos proyectos tendrán un mercado completamente seguro, en razón que el Estado ecuatoriano se compromete a comprar la energía, a través de un título habilitante que contempla un precio fijo (dependiendo del tipo de generación) que tendrá vigencia de 15 años.

Los precios por tipo de proceso de generación establecidos por el CONELEC son los siguientes:

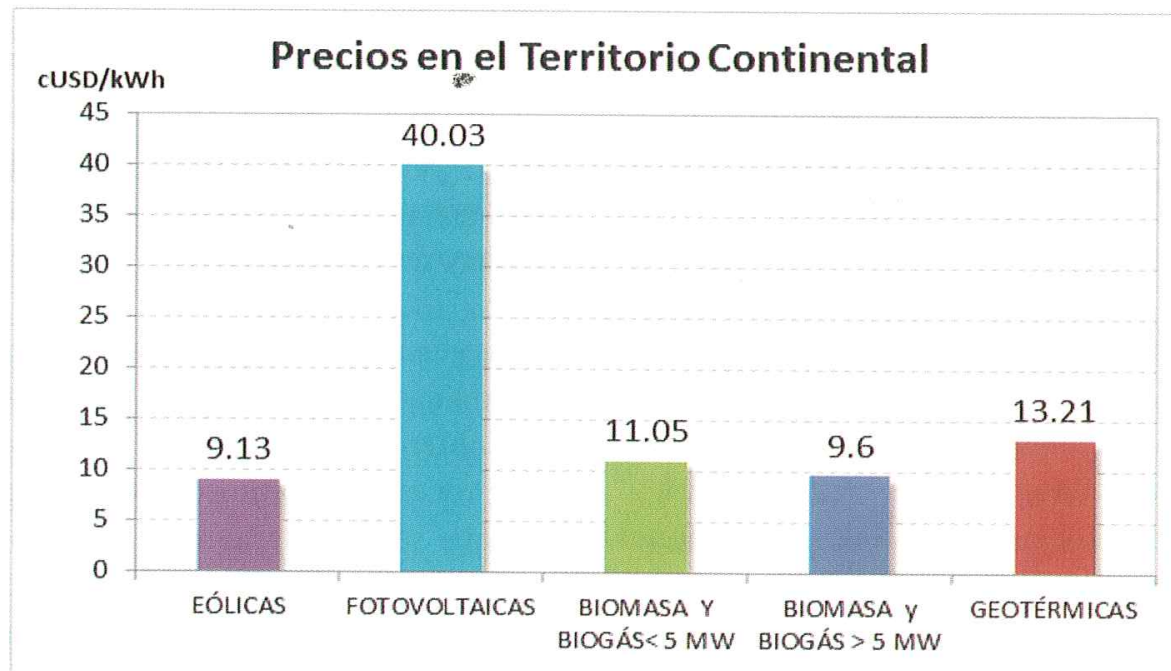

Fuente: CONELEC

Elaboración: CONELEC

La generación de energías no convencionales ha permitido un avance significativo en la construcción de equipos que permitan mejorar la eficiencia y eficacia en el aprovechamiento de recursos renovables, es así que a pesar que las inversiones son montos muy significativos si son posibles de ejecutarse.
Desde el punto de vista financiero se establece que aproximadamente por cada megavatio de generación de energía fotovoltaica se requiere una inversión de dos millones de dólares, sin embargo, los costos y gastos de operación son mínimos, lo que permite mantener índices de rentabilidad favorables, convirtiéndolos en negocios atractivos para empresarios nacionales o extranjeros. 
Con este análisis preliminar y exploratorio se establece que los proyectos de generación de energías limpias y no convencionales son negocios atractivos, tal es el caso que en el Consejo Nacional de Electricidad del Ecuador (CONELEC), ya existen algunos proyectos fotovoltaicos para su respectiva aprobación y ejecución.

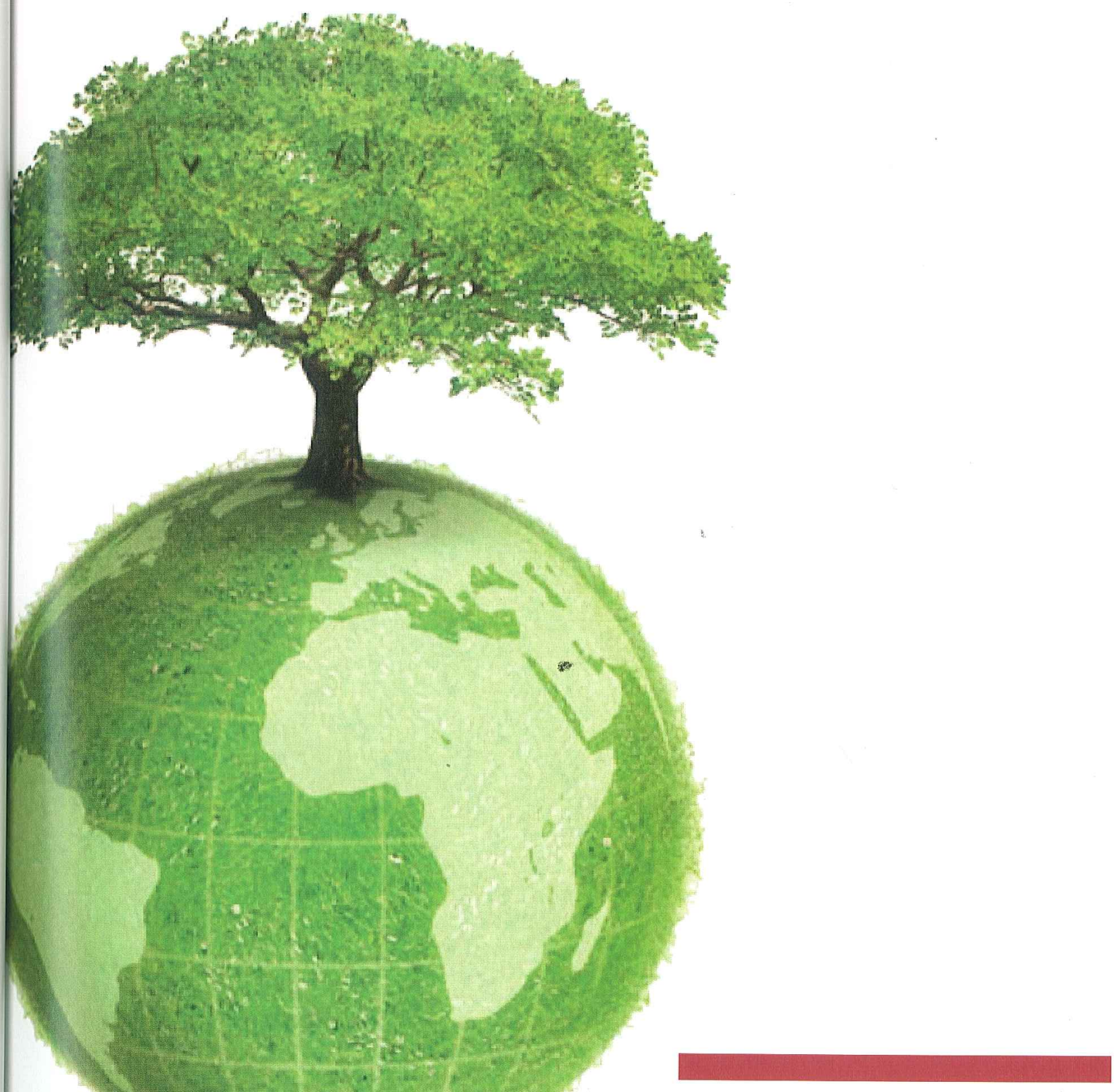

\section{Bibliografia utilizada:}

- Página Web Oficial de CONELEC

- Wikipedia, la enciclopedia libre

- http://www.bonosdecarbono.com.ar/

- El Bosque en el Ecuador por: Gerardo Barrantes, Henry Chaves,Marco Vinueza

- Página Web Oficial de FAO

- La situación de los recursos forestales - análisis regional: FAO.

- Situación Actual de la Forestación y Reforestación en el Ecuador: Tony Zúñiga Suárez - INEFAN, Ecuador. 